\title{
MENSURAÇÃO DA CURVA ESCOLIÓTICA PELA TÉCNICA DE COBB INTRAOBSERVADORES E INTEROBSERVADORES E SUA IMPORTÂNCIA CLÍNICA
}

\author{
MEASUREMENT OF THE SCOLIOTIC CURVE BY COBB TECHNIQUE FOR INTRA-OBSERVER \\ AND INTER-OBSERVER AND ITS CLINICAL SIGNIFICANCE \\ MEDICIÓN DE LA CURVA DE ESCOLIOSIS MEDIANTE LA TÉCNICA DE COBB PARA INTRAO- \\ BSERVADORES E INTEROBSERVADORES Y SU SIGNIFICACIÓN CLIINICA
}

Rigel Rego de Sá Godinho', Renato Hiroshi Salvioni Ueta², David Del Curto², Délio Eulálio Martins³, Marcelo Wajchenberg ${ }^{4}$, Eduardo Barros Puertas ${ }^{5}$

\begin{abstract}
RESUMO
Objetivo: Analisar a mensuração manual do ângulo de Cobb entre profissionais com diferentes experiências da área de Ortopedia e Traumatologia e Cirurgia da Coluna Vertebral, para observar se existem diferenças na avaliação das radiografias de pacientes portadores de escoliose idiopática intra e interobservadores, a ponto de influenciar no tratamento e seguimento desses pacientes. Métodos: Foram utilizadas para este estudo 22 radiografias simples da coluna toracolombar, póstero-anterior, de pacientes portadores de escoliose idiopática, em acompanhamento regular no ambulatório do Grupo da Coluna do Hospital São Paulo. Os exames foram avaliados por quatro diferentes categorias de profissionais ortopedistas e cirurgiões da coluna vertebral e realizada a mensuração das curvas escolióticas através do método de Cobb. As vértebras terminais das curvas não foram previamente marcadas. Somente foram mensuradas as curvas principais (consideradas as de maior valor angular) de cada radiografia. A reprodutibilidade das mensurações feitas pelos diferentes Observadores (inter-observadores) e entre as duas mensurações de cada Observador (intraobservador) foi analisada pelo Coeficiente de Correlação Intraclasse (CCl). Resultados: Foram observadas, quanto às medidas dos ângulos, concordâncias excelentes entre as avaliações intraobservadores, e observadas concordâncias excelentes entre as avaliações interobservadores, uma vez que o CCI em todas as situações manteve-se maior que 0,75, o que representa excelente reprodutibilidade. Conclusão: Concluímos que houve concordância excelente $(\mathrm{CCl}>0,75)$ na avaliação dos ângulos nas mensurações das curvas escolióticas intra e interobservadores. Ao mesmo tempo, as mensurações apresentaram variação de até $13,58^{\circ}$ intraobservadores, e de até $12,72^{\circ}$ interobservadores.
\end{abstract}

Descritores: Escoliose; Radiografia; Estudo comparativo.

\begin{abstract}
Objective: To examine the manual measurement of the Cobb angle between professionals with different experiences of Orthopedics and Traumatology and Spine Surgery, to see if there are differences in the evaluation of radiographs of patients with idiopathic scoliosis intra- and inter-observer, and it's influence in the treatment and follow-up of these patients. Methods: We used for this study, 22 radiographs of the thoracolumbar spine, posterior-anterior in patients with idiopathic scoliosis, and regularly monitored at the outpatient clinic of the Spine Group of the Hospital São Paulo. The exams were evaluated by four different categories of professional orthopedists and spine surgeons and made the measurement of scoliotic curves by the method of Cobb. End-vertebrae of the curves were not previously marked. Only the principal curves were measured (considered the highest value angle) of each radiograph. The reproducibility of measurements made by different observers (inter-observer) and between the two measurements of each observer (intra-observer) was analyzed by intraclass correlation coefficient (ICC). Results: We observed on the measures of angles, excellent concordance between assessments intra-observer and observed excellent concordance between assessments inter-observer, since the ICC at all times remained higher than 0.75, which represents excellent reproducibility. Conclusion: There was excellent concordance (ICC > 0.75) in the evaluation of measurements of angles in the scoliotic curves intra- and inter-observers. At the same time, measurements showed variations of up to $13.58^{\circ}$ intra-observer, and up to $12.72^{\circ}$ inter-observer.
\end{abstract}

Keywords: Scoliosis; Radiography; Comparative study.

\section{RESUMEN}

Objetivo: Analizar la medición manual del ángulo de Cobb entre profesionales con diferentes experiencias de Ortopedia y Traumatología y Cirugía de la Columna Vertebral, para ver si hay diferencias en la evaluación de radiografías de pacientes con escoliosis idiopática intra e interobservadores, al punto para influir en el tratamiento y el seguimiento de estos pacientes. Métodos: Se utilizaron para este estudio, 22

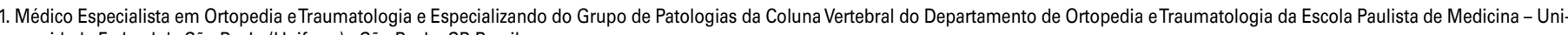
versidade Federal de São Paulo (Unifesp ) - São Paulo, SP. Brasil.

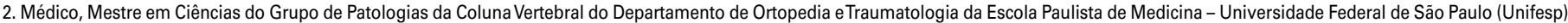
São Paulo, SP. Brasil.

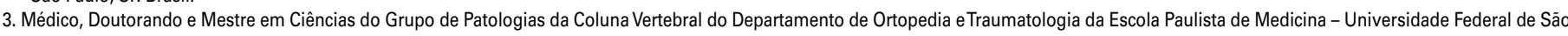
Paulo (Unifesp) - São Paulo, SP. Brasil.

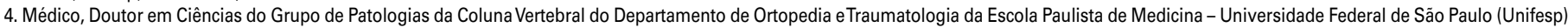
São Paulo, SP. Brasil.

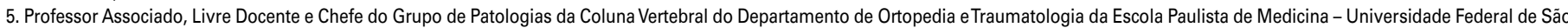
Paulo (Unifesp)- São Paulo, SP. Brasil.
} 
radiografías simples de la columna toracolumbar, posteroanterior en pacientes con escoliosis idiopática, y con seguimiento regular en la clínica de consulta externa del Grupo de Columna Vertebral del Hospital San Pablo. Los exámenes fueron evaluados por cuatro categorías diferentes de profesionales ortopedistas y cirujanos de la columna vertebral, y se hizo la medición de las curvas de escoliosis por el método de Cobb. Vértebras terminales de las curvas no fueron marcados previamente. Sólo las curvas principales se midieron (considerado el mayor ángulo de valor) en cada radiografía. La reproducibilidad de las mediciones realizadas por distintos observadores (interobservadores) y entre las dos mediciones de cada obsenador (intraobservador) fue analizada por el Coeficiente de Correlación Intraclase (CCl). Resultados: Se observó, en las medidas de los ángulos, excelentes concordancias entre las evaluaciones intraobservadores y fueron observadas excelentes concordancias entre las evaluaciones interobservadores, ya que el CCl en todas las situaciones se mantuvo superior a 0,75, lo que representa una excelente reproducibilidad. Conclusión: Se concluye en que hay un excelente acuerdo $(\mathrm{CCl}>0,75)$ en la evaluación de los ángulos en las mediciones de las curvas de escoliosis intra e interobservadores. Al mismo tiempo, las mediciones mostraron variaciones de hasta $13,58^{\circ}$ intraobservadores, y de hasta $12,72^{\circ}$.interobservadores.

\section{Descriptores: Escoliosis; Radiografía; Estudio comparativo.}

\section{INTRODUÇÃO}

A escoliose é uma deformidade com desvio lateral no plano coronal da coluna vertebral, que pode ser observado em pacientes infantis, juvenis, adolescentes e adultos, dependendo do tipo de escoliose em questão. Dentre os principais tipos, temos a escoliose idiopática, congênita, neuromuscular e escoliose degenerativa, cada uma delas predominando em uma faixa etária e com peculiaridades que as diferenciam e as caracterizam. A escoliose idiopática é caracterizada por ser uma deformidade tridimensional da coluna vertebral, pois além da curva lateral,apresenta-se com rotação vertebral, com maior predominância em indivíduos adolescentes e do sexo feminino ${ }^{1}$.

O diagnóstico e conduta tardios podem resultar em deformidades sérias, afetando a aparência física, função cardiopulmonar e bem estar psicológico. A avaliação oportuna e confiável da escoliose é essencial para a escolha do tratamento apropriado.

A avaliação da escoliose tem como ferramenta fundamental o estudo das radiografias, sendo a incidência anteroposterior o padrão para mensuração dessas curvas. Para acompanhamento e observação do quadro do paciente e avaliação da progressão da deformidade, os métodos de $\mathrm{Cobb}^{2} \mathrm{e}$ Ferguson ${ }^{3}$, que permitem quantificação da curva, são os mais comumente utilizados atualmente para mensuração da escoliose ${ }^{4,5}$, sendo o método de Cobb, particularmente, recomendado pela "Scoliosis Research Society" e amplamente usado na prática clínica ${ }^{4}$, como método "padrão ouro", para planejar o tratamento e avaliar os resultados ${ }^{6-10}$. A técnica de Cobb mede a amplitude da curva pela mensuração e cálculo do ângulo entre as linhas, respectivamente traçadas, que tangenciam a placa terminal superior da vértebra cranial e a placa terminal inferior da vértebra caudal, da curva escoliótica a ser medida ${ }^{6,11}$. Vários estudos revelam que o método de Cobb é propenso a erros e não confiável ${ }^{4,12,13}$

Avanços em tecnologia de imagem nos últimos anos tem levado a digitalização de imagens radiográficas tradicionais da coluna e a consequente melhora na análise de imagens ${ }^{4}$. No entanto, nos deparamos com estruturas ainda deficientes nos serviços públicos, aonde exames de imagens de radiografias são feitas da maneira tradicional, em filmes. Nos dias de hoje temos já vários estudos que relatam sobre a mensuração da gravidade das curvas escolióticas por meio digital e, dentre eles, alguns que comparam essas novas alternativas com a maneira manual tradicional. Porém como a tecnologia digital ainda não faz parte do dia-a-dia da vida dos ortopedistas brasileiros, e sendo a maneira de mensuração manual a nossa realidade, será esta portanto a estudada em nosso trabalho.

\section{OBJETIVOS}

Comparar a mensuração manual do ângulo de Cobb de pacientes portadores de escoliose idiopática entre profissionais com diferentes experiências da área de Ortopedia e Traumatologia e Cirurgia da Coluna Vertebral, e analisar diferenças na avaliação das radiografias intra e inter-observadores.

\section{MÉTODOS}

Foram utilizados para este estudo 22 radiografias simples da coluna toracolombar, em ortostase, na incidência póstero-anterior, de pacientes portadores de escoliose idiopática, entre cinco e 37 anos de idade, em acompanhamento regular no ambulatório do Grupo da Coluna do Departamento de Ortopedia e Traumatologia da Escola Paulista de Medicina - Universidade Federal de São Paulo. Para a obtenção dos exames, os pacientes não foram submetidos a alguma carga de radiação além daquela já necessária para o seu acompanhamento ambulatorial.

Os exames foram avaliados por quatro diferentes categorias de profissionais ortopedistas e cirurgiões da coluna vertebral: Residente de Ortopedia e Traumatologia (três anos de experiência), sendo nomeado Res-OT; Especializando em Cirurgia da Coluna Vertebral (quatro anos de experiência), nomeado como Esp-Col; Cirurgiões ortopedistas da Coluna Vertebral com seis e quinze anos de experiência, chamados de Cir-Jr. e Cir-Sênior, respectivamente.

Foi realizada a mensuração das curvas escolióticas através do método descrito por $\mathrm{Cobb}^{2}$, a qual todos os avaliadores estavam familiarizados. As vértebras terminais das curvas não foram previamente marcadas, para analisar a interpretação de cada profissional em relação a identificação das vértebras que pertencem à curva, sendo somente considerado concordantes quando o nível superior e inferior foram semelhantes. Somente foram mensuradas as curvas principais (consideradas as de maior valor angular) de cada radiografia.

Os nomes dos pacientes na identificação dos exames foram subtraídos e substituídos por etiquetas numeradas aleatoriamente (Figura 1). Foram utilizadas a mesma régua própria para medição de curva escoliótica de haste única, a mesma lapiseira e as mesmas condições para todos os participantes (Figura 2). Após a primeira medição por cada um dos profissionais, nova medição por cada um dos representantes de cada categoria foi realizada, mas com nova organização na ordem de identificação numérica dos exames e intervalo mínimo de duas semanas entre a primeira e a segunda medição, com o intuito de reduzir algum efeito de memorização.

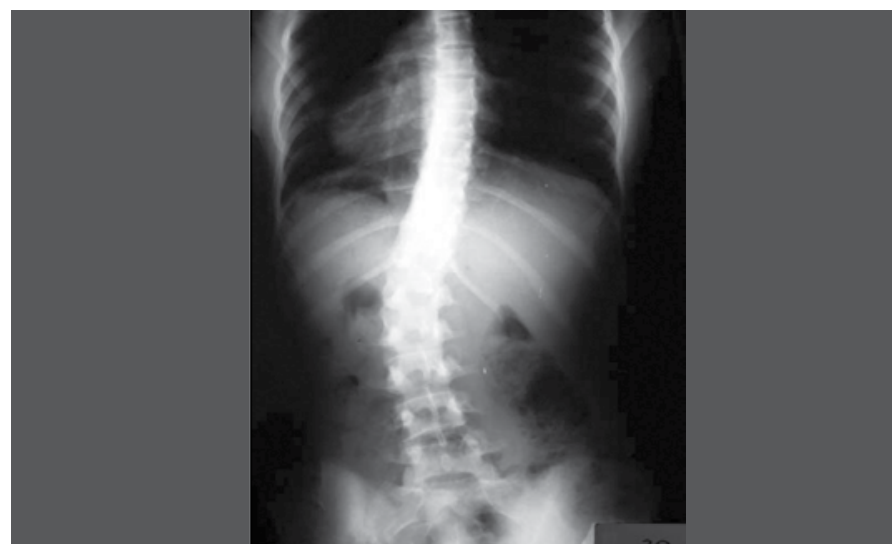

Figura 1. Exemplo de imagem radiográfica ântero-posterior utilizada para mensuração angular de coluna escoliótica. 


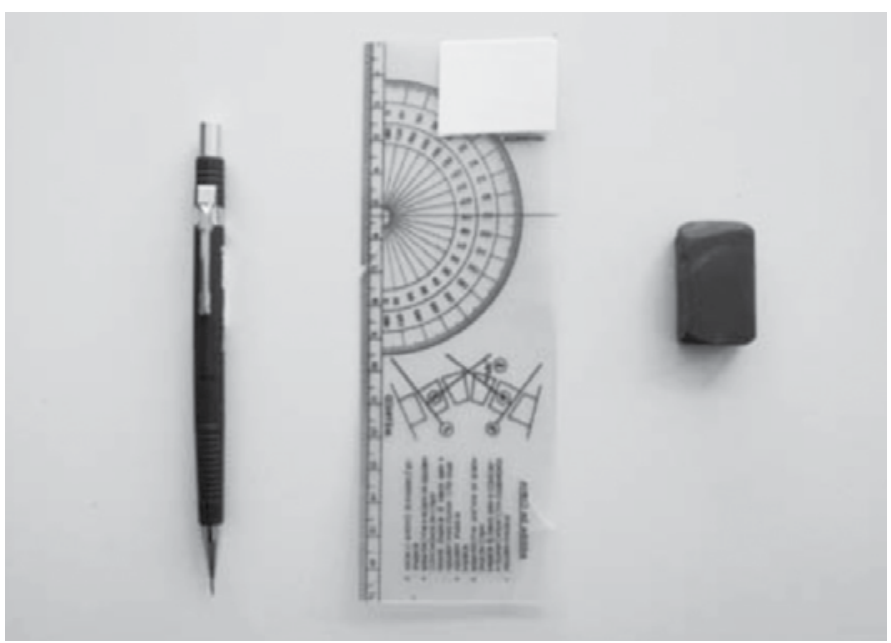

Figura 2. Material utilizado para mensuração manual.

Foram excluídos pacientes submetidos a alguma cirurgia prévia na coluna vertebral, aqueles com alguma anomalia congênita e aqueles com radiografias que apresentaram características não compatíveis com escoliose idiopática, além de paciente gestante. O estudo utilizou a infraestrutura do Hospital São Paulo, tendo o ambulatório do Grupo da Coluna do Departamento de Ortopedia e Traumatologia EPM-Unifesp como o principal local de processamento de suas etapas. Este estudo foi analisado e aprovado pelo Comitê de Ética em Pesquisa da Universidade Federal de São Paulo, sob o protocolo 0298/11.

\section{METODOLOGIA ESTATÍSTICA}

As variáveis foram resumidas e apresentadas pelas estatísticas descritivas pertinentes: média, desvio padrão (dp), mediana, valores mínimos e máximos para as variáveis quantitativas ou frequências absoluta(n) e relativa(\%) para as variáveis categóricas. Foi utilizado o teste de Kolmogorov-Smirnov para avaliar se as variáveis quantitativas contínuas seguiam uma distribuição normal.

A reprodutibilidade das mensurações feitas pelos diferentes observadores (interobservadores) e entre as 2 mensurações de cada observador (intraobservador) foi analisada pelo Coeficiente de Correlação Intraclasse $(\mathrm{CCl})^{14}$, em que valores de $\mathrm{CCl}<0,4$ indicam baixa reprodutibilidade; $0,4<\mathrm{CCl}<0,75$ indica boa reprodutibilidade; e $\mathrm{CCl}>0,75$ indica excelente reprodutibilidade.

Adotou-se o nível de significância de 0,05 (a = 5\%) em todos os testes estatísticos aplicados e níveis descritivos (p) inferiores a esse valor foram considerados estatisticamente significantes e representados por *. Todas as análises estatísticas foram executadas pelo software estatístico SPSS for Windows versão 17.0

\section{RESULTADOS}

Foram observados, quanto às medidas dos ângulos, concordâncias excelentes entre as avaliações intra-observadores $\left(1^{\mathrm{a}} \mathrm{e}\right.$ $2^{a}$ medição) (Tabela 1), e observadas concordâncias excelentes entre as avaliações interobservadores(na $1^{\mathrm{a}}$ e $2^{\mathrm{a}}$ medição), uma vez que o $\mathrm{CCl}$ em todas as situações manteve-se maior que 0,75 , que representa excelente reprodutibilidade.

Foram observadas concordâncias excelentes entre as avaliações inter-observadores (na $1^{\mathrm{a}}$ medição) quanto às medidas dos ângulos, com um $\mathrm{CCl}=0,945$.

Em relação as medidas intra-observadores das curvas mensuradas, notamos grande variação nos valores obtidos pelo observador especializando em coluna, mostrando desvio padrão(13,58 $)$. Por outro lado, a menor variação foi observada quando avaliados os valores medidos pelo residente em ortopedia, com desvio padrão de $5,29^{\circ}$ (Tabela 2), seguido pelo cirurgião sênior, com variação de $6,67^{\circ}$.
Tabela 1. Concordância intra-observador quanto às medições dos ângulos.

\begin{tabular}{c|c|c|c}
\hline \multicolumn{4}{c}{ Observador } \\
\hline RES-0T & ESP-COL & CIR-JR. & CIR-SENIOR \\
\hline $\mathrm{CCl}=0,982$ & $\mathrm{CCl}=0,907$ & $\mathrm{CCl}=0,966$ \\
$\mathrm{p}<0,001^{*}$ & $\mathrm{p}<0,001^{*}$ & $\mathrm{p}<0,001^{*}$ & $\begin{array}{l}\mathrm{CCl}=0,975 \\
\mathrm{p}<0,001^{*}\end{array}$ \\
\hline
\end{tabular}

*níveis descritivos $<0,05(p<0,05)$

Tabela 2. Mensuração intra-observadores dos ângulos pela técnica de Cobb (em graus)

\begin{tabular}{c|c|c|c|c}
\hline Observadores & Res-0t & Esp-Col. & Cir-Jr. & Cir-Senior \\
\hline Média & $-2,82$ & 0,86 & 1,45 & $-0,32$ \\
\hline Dp & 5,29 & 13,58 & 8,19 & 6,67 \\
\hline Dp-desvio padrão
\end{tabular}

Ao analisarmos as medidas inter-observadores notamos que a maior variação ocorreu quando as curvas foram medidas pelo residente em ortopedia, comparado com o especializando em cirurgia de coluna, com desvio padrão de $12,72^{\circ}$. No entanto, observamos que a variação entre os avaliadores foi próxima a $10^{\circ}$ (Tabela 3).

Ao observarmos a concordância dos níveis mensurados intraobservadores, percebemos maiores concordâncias entre os observadores cirurgião sênior e residente em Ortopedia, com valores de $63,9 \%$ e $59 \%$, respectivamente (Tabela 4 ).

Quando analisado a concordância inter-observadores dos níveis mensurados, observa-se uma maior porcentagem quando comparados o residente em Ortopedia e o cirurgião de coluna sênior, de 54,5\%; e uma menor concordância entre o residente em ortopedia e o cirurgião de coluna júnior, de 22,7\% (Tabela 5).

As medidas individuais dos ângulos, de cada radiografia dos os pacientes envolvidos nesse estudo, estão ilustradas na Figura 3, o qual mostra os resultados obtidos por todos os observadores, tanto na $1^{\mathrm{a}}$ quanto na $2^{\mathrm{a}}$ medição.

Tabela 3. Mensuração dos ângulos inter-observadores pela técnica de Cobb (em graus).

\begin{tabular}{c|c|c|c|c|c|c}
\hline & $\mathbf{R O} \underline{\mathbf{X}} \mathrm{EC}$ & $\mathbf{R O} \underline{\mathbf{X}} \mathbf{C J}$ & $\mathbf{R O} \underline{\mathbf{X}} \mathbf{C S}$ & $\mathbf{E C} \underline{\mathbf{X} C \mathbf{J}}$ & $\mathbf{E C} \underline{\mathbf{X}} \mathbf{C S}$ & $\mathbf{C J} \underline{\mathbf{X}} \mathbf{C S}$ \\
\hline Média & $-2,14$ & $-2,86$ & $-1,82$ & $-0,73$ & 0,32 & 1,05 \\
\hline Dp & 12,72 & 7,49 & 10,48 & 10,47 & 10,69 & 9,24 \\
\hline
\end{tabular}

RO - Residente em Ortopedia e Traumatologia. EC - Especializando em Coluna. CJ Cirurgião Júnior. CS - Cirurgião Sênior. X-Versus.

Tabela 4. Concordância dos níveis mensurados intra-observadores.

\begin{tabular}{c|c|c|c|c}
\hline Observadores & Res-Ot & Esp-Col. & Cir-Jr. & Cir-SÊNior \\
\hline Concordância (\%) & 59 & 45,5 & 40,9 & 63,9 \\
\hline
\end{tabular}

Tabela 5. Concordância dos níveis mensurados inter-observadores.

\begin{tabular}{c|c|c|c|c|c|c}
\hline & RO $\underline{x}$ EC & RO $\underline{x}$ CJ & RO $\underline{x}$ CS & EC $\underline{x}$ CJ & EC $\underline{x}$ CS & CJ $\underline{x}$ CS \\
\hline Concordância (\%) & 45,5 & 22,7 & 54,5 & 27,3 & 27,3 & 27,3 \\
\hline
\end{tabular}

Cirurgião Júnior. CS - Cirurgião Sênior. X - Versus. 


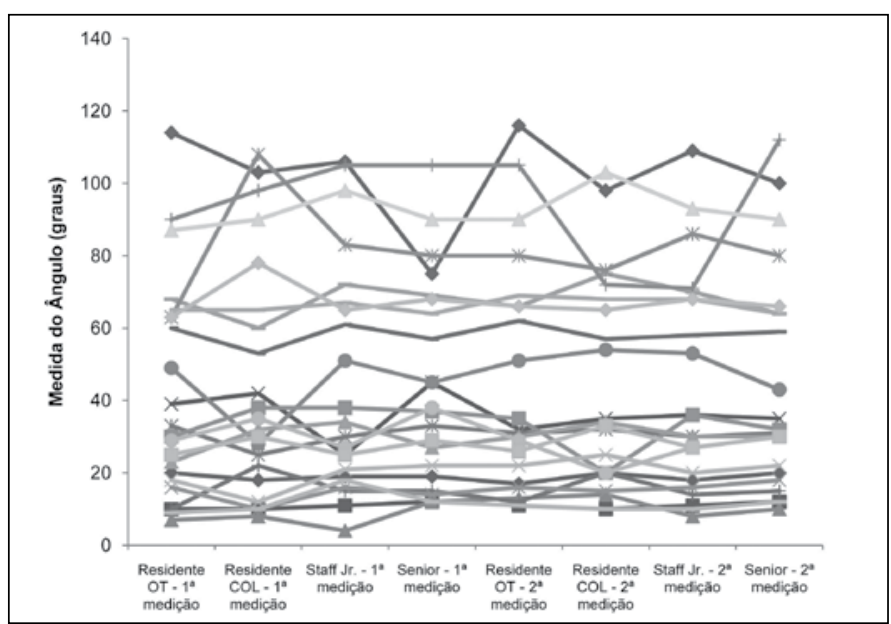

Figura 3. Medidas individuais dos ângulos pela técnica de Cobb nas diferentes medições.

\section{DISCUSSÃO}

Muitos estudos avaliaram variações nas mensurações de curvas escolióticas pelo método de Cobb, tanto intra-observadores e interobservadores ${ }^{4-17}$. A mensuração manual do ângulo de Cobb depende da experiência e julgamento do profissional que está fazendo a medição ${ }^{11}$. A seleção da vértebra terminal, tanto cranial quanto caudalmente, pertencentes à curva, enquadra-se como importante parâmetro para a mensuração da curva escoliótica idiopática ${ }^{5-10,15}$. Devido a influência que esta seleção representa para determinar o valor da curva na escoliose idiopática, no nosso estudo avaliamos a concordância intra e inter-observador em relação aos níveis mensurados.

Observou-se no nosso estudo concordâncias excelentes entre as avaliações inter e intra-observadores, mesmo com todas as adversidades existentes relacionadas à mensuração manual4,8,9,11,12,15. Podemos ressaltar dados interessantes no que diz respeito tanto à porcentagem de concordância intra e inter-observadores em relação aos níveis incluídos na curva escoliótica, quanto à variação em graus das mensurações das mesmas.

Observamos concordância para a escolha dos níveis de 59\% apresentada pelo residente em Ortopedia na análise intra-observacional, menor apenas que a concordância do cirurgião sênior, de $63,9 \%$, dentre todos os quatro profissionais envolvidos. Chama a atenção o fato de o menos experiente dos observadores ter obtido resultados mais concordantes que o especializando em coluna e do que o cirurgião Júnior. Esta situação pode ser explicada pela maior cautela de um profissional menos familiarizado com a técnica que irá empregar para a mensuração do exame, o que pode levar a obtenção de melhores resultados do que de outro profissional mais experiente que, por ter supostamente mais intimidade com a técnica, pode aplicá-la de maneira menos atenciosa. Quando analisamos as concordâncias inter-observadores dos níveis escolhidos, observamos que a maior porcentagem é justamente entre os dois observadores com maior concordância no estudo intra-observacional, ou seja, o residente em ortopedia e o cirurgião sênior, que apresentam uma concordância dos níveis vertebrais de $54,5 \%$.

$\mathrm{Na}$ análise da mensuração, em graus, das curvas no estudo intra-observacional, a variação obtida pelo residente em Ortopedia foi nessa ocasião a melhor, sendo 5,29, seguida pelo cirurgião sênior, que obteve um valor de 6,67\%. Dados esses que podemos relacionar com o estudo das concordâncias dos níveis, uma vez que o resultado angular obtido numa mensuração de curva escoliótica depende dos níveis selecionados, ou seja, da seleção das vértebras terminais das extremidades cranial e caudal pertencentes à curva a ser medida $8,9,11,12$. Uma vez que observamos os mesmos dois profissionais com as melhores concordâncias intra e interobservadores (Res-OT e Cir-Senior), observamos a influência da escolha dos níveis com os valores angulares obtidos das curvas, relacionados com os menores valores de desvio padrão na análise intra-observacional, também para os dois mesmos observadores. Todavia, na análise inter-observadores das mensurações angulares, temos um menor desvio padrão entre o residente em Ortopedia e o cirurgião Júnior, de 7,49o. Em outro recente estudo nacional ${ }^{13}$ que também avaliou a reprodutibilidade intra e inter-observadores em indivíduos com escoliose idiopática, pelo método manual de Cobb, foi relatado diferenças de até $3^{\circ}$ intra-observador. Foi evidenciado ainda neste estudo citado, uma concordância intra-observador para o ângulo de Cobb com relação significativa excelente, independente do nível da curva, sendo que, na concordância inter-observador, houve relação estatisticamente significativa no nível torácico, mas no nível lombar os observadores não apresentaram correlação significativa entre si.

Tradicionalmente, mesmo aceitando variação de $5^{\circ}$ como valor necessário para confirmar a evolução da curva ${ }^{9}$, esses valores tem sido contestados por alguns estudos, uma vez que valores como $95 \%$ dos intervalos de confiança (ICs) com variação de $2.6^{\circ}$ a $8.8^{\circ}$ já foram relatados ${ }^{9}$. No nosso estudo, observou-se variações de até $12,72^{\circ}$ de desvio padrão.

Muitos estudos revelam o método de Cobb como propenso a erros e como não confiável ${ }^{1}$. Erros são devido à seleção de diferentes vértebras terminais ("end vertebras") $8,9,11,12$ e à estimativa de inclinações diferentes das vértebras ${ }^{8,11,12}$. Em estudos, os quais as vértebras incluídas nas curvas escolióticas foram pré determinadas, foram observados desvio padrão de mensuração além de $5^{\circ 11}$. Outro fator que pode proporcionar diferenças na mensuração da curva da coluna vertebral é a escolha das referências ósseas para empregar o método de Cobb: platôs vertebrais ou pedículos ${ }^{6}$, os quais, segundo Mehta et al. ${ }^{6}$,variam como opção de acordo com a idade do paciente, sendo o método de mensuração com os pedículos ligeiramente melhor para crianças menores que sete anos de idade, e similar, se não melhor, para os outros grupos, quando comparado ao método de mensuração com as placas terminais (platôs). Loder et al. ${ }^{16}$ relata que dificuldades para identificação da placa terminal para mensuração angular são ainda maiores nos casos das escolioses congênitas, devido a imaturidade esquelética, ossificação incompleta e desenvolvimento anômalo das vértebras terminais. A confiabilidade dos parâmetros radiográficos nas escolioses neuromusculares foi estudada por Gupta et al. ${ }^{17}$, que relata o método de Cobb como método também seguro para esse tipo de deformidade. No nosso estudo, a escolha da referência utilizada para mensuração da curva escoliótica foi de livre escolha para os observadores.

Temos como parâmetros para tratamento da escoliose idiopática, deformidade na coluna com valores angulares que quando entre $10^{\circ}$ e $25^{\circ}$ será monitorada regularmente até a maturidade esquelética ou progressão significativa da curva; valores entre $25^{\circ} \mathrm{e}$ $45^{\circ}$, o tratamento com órtese (brace) é sugerido; valor é maior que $45^{\circ}$, o tratamento cirúrgico geralmente é recomendado ${ }^{11}$.Considerando que, na prática clínica, é indicação de progressão da curva a variação superior a cinco graus entre duas radiografias sucessivas, num intervalo de 6 meses ${ }^{10,11,12}$, faz-se necessário análise cautelosa, associado à experiência do observador, e que, preferencialmente, os exames sejam avaliados pelo mesmo profissional durante o acompanhamento de pacientes portadores de escoliose idiopática, para que as condutas sejam tomadas de maneira mais fidedigna.

Visto a extrema importância desse método de mensuração do tamanho da curva escoliótica, diretamente relacionada ao quadro clínico e tratamento ${ }^{4,11,12,15}$, atentamos ao fato de aprofundar a discussão sobre a sua confiabilidade no nosso meio.

Outro fator importante ressaltado em alguns estudos referese a uma das maiores limitações do método de Cobb, que é a 
representação da deformidade tri-dimensional (3D) complexa da coluna escoliótica por uma mensuração simples em duas dimensões (2D), o que não permite uma descrição completa e fidedigna de toda extensão da curva, sendo de difícil obtenção informações sobre os três planos (coronal, sagital e transverso) na prática clínica ${ }^{13,15}$. Gstoettner et al. ${ }^{8}$ concluíram que, até o surgimento de um sistema de mensuração próprio tri-dimensional, o método de Cobb é capaz somente de avaliar a curva escoliótica em dois planos, por melhor que seja a qualidade da imagem em AP e no perfil. Com o aparecimento dos métodos de mensuração através de imagens digitalizadas e também através do computador, muitos trabalhos foram feitos com o intuito de comparar a acurácia desses novos sistemas com o sistema convencional de mensuração. Alguns desses estudos concluem que não há diferenças significativas com relação aos resultados, quando comparados os sistemas de mensuração manual, de imagens digitalizadas e ou pelo computador ${ }^{8-10}$. Outros artigos, no entanto, concluem que os sistemas de mensuração de imagens digitais, e ou com auxílio do computador, apresentam uma variabilidade intra e inter- observadores muito menor quando comparados aos sistemas de mensuração manual $\left.\right|^{5,15}$.

Neste estudo, procuramos reproduzir a rotina no atendimento aos pacientes portadores de escoliose idiopática. Considerando que grande parte destes pacientes são acompanhados em centros universitários, muitos desses na rede pública de saúde, nos quais são atendidos por profissionais de diferentes níveis de experiência e dispondo, por muitas vezes, de radiografias simples. Apesar do valor de concordância ter sido considerado excelente em todas as mensurações $(\mathrm{CCl}>0,75)$, feitas nas condições relatadas, observamos que houve variação importante das medidas em graus com relação à avaliação intra e interobservadores, com valores de até $13,58^{\circ} \mathrm{e}$ $12,72^{\circ}$, respectivamente. Por outro lado, menores variações foram registradas quando a curva escoliótica foi medida por um mesmo avaliador (residente em Ortopedia e cirurgião Sênior). Ressaltando, portanto, a importância da análise cautelosa e também a experiência do observador na mensuração das curvas escolióticas.

\section{CONCLUSÃO}

Concluímos que houve concordância excelente $(\mathrm{CCl}>0,75)$ na avaliação dos ângulos nas mensurações das curvas escolióticas intra e inter-observadores pelo método manual de Cobb. Ao mesmo tempo, as mensurações apresentaram variação de até $13,58^{\circ}$ intraobservadores, e de até $12,72^{\circ}$ interobservadores.

\section{REFERÊNCIAS}

1. Bunnell WP. The natural history of idiopathic scoliosis. Clin Orthop Relat Res. 1988;(229):20-5

2. Cobb J. Outline for the study of scoliosis. Instr Course Lect. 1948;5:261.

3. Ferguson AB. The study and treatment of scoliosis. South Med J., Birmingham, 1930; 23(2):116-120

4. He JW, Yan ZH, Liu J, Yu ZK, Wang XY, Bai GH, et al. Accuracy and repeatability of a new method for measuring scoliosis curvature. Spine (Phila Pa 1976). 2009:34(9):E323-9.

5. Srinivasalu S, Modi HN, Smehta S, Suh SW, Chen T, Murun T. Cobb angle measurement of scoliosis using computer measurement of digitally acquired radiographs-intraobserver and interobserver variability. Asian Spine J. 2008;2(2):90-3.

6. Mehta SS, Modi HN, Srinivasalu S, Chen T, Suh SW, Yang JH, et al. Interobserver and intraobserver reliability of Cobb angle measurement: endplate versus pedicle as bony landmarks for measurement: a statistical analysis. J Pediatr Orthop. 2009:29(7):749-54

7. De Carvalho A, Vialle R, Thomsen L, Amzallag J, Cluzel G, le Pointe HD, et al. Reliability analysis for manual measurement of coronal plane deformity in adolescent scoliosis. Are $30 \times 90 \mathrm{~cm}$ plain films better than digitized small films? Eur Spine J. 2007:16(10):1615-20

8. Gstoettner M, Sekyra K, Walochnik N, Winter P, Wachter R, Bach CM. Inter- and intraobserver reliability assessment of the Cobb angle: manual versus digital measurement tools. Eur Spine J. 2007;16(10):1587-92.

9. Wills BP, Auerbach JD, Zhu X, Caird MS, Horn BD, Flynn JM, et al. Comparison of Cobb angle measurement of scoliosis radiographs with preselected end vertebrae: traditional versus digital acquisition. Spine (Phila Pa 1976). 2007;32(1):98-105.

10. Mok JM, Berven SH, Diab M, Hackbarth M, Hu SS, Deviren V. Comparison of observer variation in conventional and three digital radiographic methods used in the evaluation of patients with adolescent idiopathic scoliosis. Spine (Phila Pa 1976). 2008:33(6):681-6.

11. Zhang J, Lou E, Le LH, Hill DL, Raso JV, Wang Y. Automatic Cobb measurement of scoliosis based on fuzzy Hough Transform with vertebral shape prior. J Digit Imaging. 2009;22(5):463-72.

12. Allen $\mathrm{S}$, Parent $\mathrm{E}$, Khorasani $\mathrm{M}$, Hill $\mathrm{DL}$, Lou $\mathrm{E}$, Raso JV. Validity and reliability of active shape models for the estimation of cobb angle in patients with adolescent idiopathic scoliosis. J Digit Imaging. 2008;21(2):208-18.

13. Sperandio FF, Tavares GMS, Santos GM. Reprodutibilidade intra e interobservadores no ângulo de Cobb em indivíduos escolióticos. Rev Bras Cresc. Desenv. Hum. $2011 ; 21(1): 21-9$.

14. Fleiss JL. The design and analysis of clinical experiments. New York: John Wiley \& Sons; 1986.

15. Cheung J, Wever DJ, Veldhuizen AG, Klein JP, Verdonck B, Nijlunsing R, et al. The reliability of quantitative analysis on digital images of the scoliotic spine. Eur Spine J. 2002:11(6):535-42.

16. Loder RT, Urquhart A, Steen H, Graziano G, Hensinger RN, Schlesinger A, et al. Variability in Cobb angle measurements in children with congenital scoliosis. J Bone Joint Surg Br. 1995:77(5):768-70.

17. Gupta MC, Wijesekera S, Sossan A, Martin L, Vogel LC, Boakes JL, et al. Reliability of radiographic parameters in neuromuscular scoliosis. Spine (Phila Pa 1976). 2007;32(6):691-5 